pag

Business School

WORKING PAPER SERIES

Working Paper

$2014-410$

\section{Optimisation d'une stratégie anti-OPA} par limitation linéaire des droits de vote face à un investisseur hostile

Bruno-Laurent Moschetto

Frédéric Teulon

http://www.ipag.fr/fr/accueil/la-recherche/publications-WP.html

IPAG Business School

184, Boulevard Saint-Germain

75006 Paris

France 


\title{
Optimisation d'une stratégie anti-OPA par limitation linéaire des droits de vote face à un investisseur hostile
}

\author{
Bruno-Laurent Moschetto* \\ Frédéric Teulon
}

Cet article s'intéresse aux mesures défensives reposant sur une limitation linéaire des droits de vote, adoptées principalement pour contrer les offres publiques d'achat hostiles. Nous étudions la situation d'une firme dont l'actionnariat est atomisé et dont le noyau dur d'actionnaires souhaite empêcher l'arrivée d'un nouvel entrant. Ce groupe met en place un dispositif de défense qui repose sur un seuil de limitation au-dessus duquel tout droit de vote est annulé puis redistribué linéairement selon un certain coefficient. Nous montrons que ce type de dispositif est efficace pour contrer tout investisseur hostile agissant seul. Cependant, ce résultat est généralement obtenu avec un paramétrage qui adresse un mauvais signal au marché en termes de gouvernance. L'article propose alors de déterminer, quand il existe, le paramétrage optimum du dispositif.

THE LINEAR VOTING RULE LIMITATION STRATEGY TO REDUCE THE POWER OF A UNIQUE NEWCOMER OVER A FIRM'S CAPITAL

This paper studies the rules that limit voting rights as a defensive measure against hostile takeovers. We consider atomistic shareholders and a small group of united minority shareholders, the hard core, that wants to block any hostile takeovers. This group constructs a device based on a threshold below which each share is linked to one vote and above which each share gives less than one vote, according to a coefficient of redistribution. It finds the optimal couple that blocks hostile takeovers while minimizing the negative impact on the market due to too strong protection of the hard core to the detriment of dispersed shareholders.

Classification JEL : G18, G30, G34

\footnotetext{
* IDP, Université de Valenciennes, France (bruno-laurent.moschetto@univ-valenciennes.fr)

** IPAG Lab, IPAG Business School, Paris, France (f.teulon@ipag.fr)
} 


\section{INTRODUCTION}

De toutes les mesures de défense contre les offres publiques d'achat, celles reposant sur des règles limitant les droits de vote sont généralement citées comme les plus efficaces. Paradoxalement, ce type de mesure a été peu étudié, testé ou modélisé. C'est précisément l'objet de notre article qui s'intéresse à l'efficacité théorique d'un tel dispositif mis en place pour contrer une OPA lancée par un unique investisseur hostile. S'il est facile de montrer que la limitation des droits de vote permet de combattre efficacement toute prise de contrôle hostile, ce résultat est généralement obtenu avec un réglage qui envoie un mauvais signal au marché en termes de gouvernance. Aussi, face à toute situation supposée hostile, notre article propose de déterminer la configuration optimale du dispositif. Idéalement, cette configuration doit tout à la fois empêcher le transfert du contrôle, maintenir la confiance du marché et permettre la tenue des assemblées générales dans de bonnes conditions.

Bien que la littérature sur les défenses anti-OPA soit abondante de nombreux axes de recherche restent ouverts. Un premier axe concerne la construction de modèles formels permettant aux gestionnaires d'une entreprise qui cherchent à contrer une OPA hostile de combiner de manière optimale les différents moyens d'action qui sont à leur disposition ${ }^{1}$. Selon Barry et Hatfield [2012]: «Une grande partie du désaccord entourant les stratégies anti-OPA provient de l'absence d'un cadre d'analyse formelle pleinement développé pour apprécier leurs effets (p. 634)». En outre, les quelques modèles existants concernent un nombre restreint de situations, ce qui limite leur portée et leur applicabilité. Par exemple, Shleifer et Vishny [1986 b] analysent des interactions liées à un chantage à l'OPA, tandis que Bagnoli, Gordon et Lipman [1989] construisent un modèle dans lequel le manager est amené à donner des informations sur la valeur de l'entreprise au fur et à mesure du déroulement de l'OPA. Quelques années plus tard, Austen-Smith et O'Brien [1992] se sont intéressés à des opérations défensives qui nécessitent l'approbation des actionnaires et Molin [1996] a analysé l'impact des changements dans les seuils de contrôle. Ce dernier montre que les pilules empoisonnées sont optimales dans les configurations de paramètres qui renvoient à une très forte probabilité de prise de contrôle, en accord avec l'évidence empirique. Plus récemment, Goldman et Qian [2005] ont calculé le nombre optimal d'actions qu'un investisseur doit acquérir avant de lancer une offre publique d'achat; ils proposent également une explication des raisons pour lesquelles les raiders n'acquièrent pas nécessairement un nombre élevé de titres avant d'annoncer une offre publique d'achat. Notre article s'inscrit pleinement dans ce premier axe et propose la construction d'un modèle spécifique aux mesures défensives liées à la limitation des droits de vote. Il est bâti à partir d'un ensemble d'hypothèses qui peuvent servir de références pour toutes les recherches sur ce sujet et pour toutes les combinaisons particulières de paramètres.

Un deuxième axe de recherche concerne l'impact des mesures de défense sur le marché. Il apparaît que l'évaluation des coûts et des avantages des systèmes de protection anti-OPA est délicate (Bebchuk et Cohen, [2005]). Turk, Goh et Ybarra [2007] suggèrent que si les mesures anti-OPA ne conduisent pas à une révision à la baisse de la prévision de bénéfice au moment où elles sont adoptées, elles sont sans

\footnotetext{
${ }^{1}$ Cela devrait se faire idéalement sans porter atteinte aux intérêts des actionnaires minoritaires.
} 
doute une réponse à leur déclin antérieur. En outre, il semble qu'il y ait souvent, mais pas toujours, une réaction défavorable significative des marchés financiers à l'adoption de ce type de mesures. Cela est également vrai en ce qui concerne le jugement des analystes (Davidson et al. [2004]; Johnson et Rao [1997]). En ce qui concerne ce deuxième axe, notre article se place dans le courant majoritaire et estime sans équivoque que l'adoption de mesures défensives est perçue négativement par le marché et que sa défiance est d'autant plus grande que le dispositif est brutal.

Un troisième axe de recherche concerne l'interaction entre les mesures de défense et la gouvernance. Sur ce point, la plupart des études empiriques concernent les entreprises américaines et intègrent peu les entreprises européennes ou asiatiques (Gompers et al [2003]; Hwang et Kim [2011]). Dans une étude de près de 500 entreprises, Rechner, Sundaramurphy et Dalton [1993] montrent que le lien entre la gouvernance d'entreprise et l'existence de mesures anti-OPA donne naissance à deux comportements très différents face au risque d'une attaque externe. Ceci est cohérent avec l'étude d'Agrawal et Mandelker [1990], auteurs qui mettent en évidence un lien entre le type de gouvernance, la répartition des droits de propriété et l'adoption de mesures anti-OPA. Plusieurs études montrent ainsi que les gestionnaires qui adoptent des mesures visant à modifier les droits de vote détiennent une part plus petite du capital de leur entreprise que ceux qui n'utilisent pas cette option. Certaines études concluent que ces mesures réduisent la probabilité d'une OPA (Bebchuk, Coates et Subramanian [2002]). D'autres études (Comment et Schwert [1995]; Heron et Lie [2006]) sont moins affirmatives et estiment que même si les mesures qui ont un impact sur les droits de vote semblent réduire les chances de succès d'une OPA, elles ne jouent pas vraiment un rôle préventif. Dans la plupart des travaux de recherche qui traitent des restructurations du capital des firmes (Jensen [1988]; Tirole [2006]), les prises de contrôle sont généralement présentées comme un outil utile pour prévenir la direction de s'enraciner et comme le seul outil de contrôle externe dans les entreprises au capital dispersé. Cependant, la plupart des équipes de gestion restent opposées à tout changement brusque de contrôle (Shleifer et Vishny [1986 a]) ce qui justifie, par conséquent, l'adoption de mesures de défense contre les OPA hostiles. De manière synthétique, si les stratégies de défense basées sur la limitation des droits de vote sont réputées efficaces, elles sont aussi souvent critiquées et il est généralement considéré que les droits de vote proportionnels à la répartition du capital social conduisent à une meilleure gouvernance ${ }^{2}$ et une plus grande discipline de gestion ${ }^{3}$.

Bien que cela soit très intéressant d'un point de vue académique, notre article ne vise pas à tester le caractère préventif du système de défense basé sur la limitation des droits de vote; son objectif est d'identifier le paramétrage le plus efficace face à une OPA hostile, initiée par un investisseur unique et visant une entreprise ayant un noyau dur d'actionnaires uni, mais non majoritaire. Le papier souhaite rester complètement neutre par rapport à la question d'une éventuelle destruction de valeur pour les actionnaires. Si nos arguments semblent favoriser la défense des actionnaires en place au détriment de l'investisseur, c'est tout simplement en raison

\footnotetext{
${ }^{2}$ En particulier, nous pouvons citer Bebchuk et al. [2009], Grossman et Hart [1988], Harris et Raviv [1988] et Jensen [1986].

${ }^{3}$ Voir également Amstrong et al. [1994], DeAngelo et Rice [1983] et Johnson et Rao [1997].
} 
de l'orientation qui a été donnée à cette recherche et à la volonté de ne pas mélanger les points de vue dans un souci de clarté.

Ce papier est structuré en sept sections. A la suite de l'introduction, la section 2 présente le cadre initial de l'étude. La section 3 met en évidence l'existence d'un seuil qui garantit l'efficacité de la stratégie de blocage de l'OPA, seuil que nous utilisons comme une fonction cible pour deux programmes d'optimisation. La section 4 décrit le premier programme d'optimisation, celui qui détermine le meilleur ajustement possible des paramètres, dans un cadre qui ne tient pas compte des conditions relatives à la tenue d'assemblées générales. Nous montrons ici qu'il est possible de concevoir un programme qui soit systématiquement efficace contre toute prise de contrôle hostile, mais avec le risque d'envoyer un signal très négatif aux marchés financiers en termes de gouvernance. Dans la même section, nous recherchons le meilleur ajustement des variables sur lesquelles la direction de l'entreprise peut agir, au travers de mesures qui vont décourager tout investisseur hostile mais qui permettront à l'entreprise de conserver la confiance du marché ${ }^{4}$. La section 5 prolonge cette ligne directrice avec un deuxième programme d'optimisation, incluant cette fois les conditions requises pour la tenue des assemblées générales. Elle étudie l'impact de cette nouvelle contrainte sur l'ajustement du modèle. Si les sections 2 à 5 décrivent le modèle dans un cadre déterministe simple dans lequel l'OPA a lieu avec certitude, la section 6 se place en dehors de ce cadre. Elle ouvre la voie à un modèle plus complexe dans lequel le déclenchement d'une prise de contrôle obéit à une loi de probabilité. La section 7 synthétise les principales contributions du papier et discute des prolongements possibles du modèle.

\section{LE CADRE INITIAL DE L'ETUDE}

Nous considérons une entreprise cotée dont le capital est constitué de T titres. Elle est la cible d'une OPA hostile pendant laquelle aucune augmentation de capital n'est possible et $\mathrm{T}$ doit donc être considéré comme une constante. Il existe trois différents profils d'actionnaires : un petit groupe d'actionnaires soudés mais minoritaires qui constituent le noyau dur, un grand nombre d'actionnaires passifs et atomisés et un nouvel entrant unique, investisseur hostile au noyau dur.

Le noyau dur se compose de $\mathrm{N}$ actionnaires, classés par ordre décroissant du nombre de titres possédés. L'actionnaire $j$ possède $l_{j}$ titres. Ensemble, les actionnaires du noyau dur possèdent A titres, nombre toujours considéré comme inférieur à la moitié du nombre total de titres émis. Ils prennent part systématiquement aux assemblées générales. Notre article s'inscrit volontairement dans un cadre contraint qui, en particulier, interdit tout changement de la structure de l'actionnariat au sein du noyau dur pendant toute la durée de l'OPA.

Les actionnaires passifs et fragmentés possèdent, ensemble, la majorité des titres, mais chacun en possède individuellement un très petit nombre. Leur action est uniquement guidée par des considérations pécuniaires et pour cette raison ils n'assistent jamais aux assemblées générales. Ces petits actionnaires agissent en free riders. Nous n'entrons pas dans cet article dans le riche débat sur le prix d'équilibre

\footnotetext{
${ }^{4}$ Dans le but d'attirer de nouveaux investisseurs minoritaires.
} 
ou sur le phénomène de surenchère $e^{5}$ et nous postulerons que le prix proposé pour le rachat d'actions lors de l'OPA est suffisamment attractif pour convaincre de nombreux actionnaires passifs de vendre leurs titres.

Dans la suite, l'investisseur hostile sera noté $h$. Dans le modèle initial, qui concerne les sections 2 à 5, l'arrivée de l'investisseur hostile est supposée certaine ${ }^{6}$. L'investisseur $h$ agit seul, dans le cadre d'une action à caractère spéculatif. Il cherche à obtenir le contrôle de l'entreprise en rachetant, au final, un nombre de titres lui garantissant de détenir plus de la moitié des droits de vote lors des assemblées générales. A la fin de l'OPA, il détient $l_{h}$ titres, nombre supposé supérieur au total des titres détenus par l'ensemble des membres du noyau dur. Cela constitue une condition nécessaire du contrôle puisque que nous avons supposé qu'aucun des membres du noyau dur ne pouvait faire sécession lors de l'opération.

L'entreprise dispose d'un système de défense anti-OPA qui repose sur le principe de la limitation des droits de vote. Ce type de défense est évidemment dépendant du cadre législatif dans lequel il s'applique (La Porta et al. [1998]) et le système que nous considérons ici est le moins restrictif possible du point de vue juridique. Le système est mis en place à l'initiative des membres du noyau dur qui souhaitent protéger le contrôle qu'ils ont sur la firme. Le système consiste tout d'abord à fixer un nombre de titre, appelé le seuil de limitation et noté $\delta$. En dessous de ce seuil, chaque action possède un droit de vote ${ }^{7}$. Au-dessus de ce seuil, les droits de vote sont annulés puis redistribués par application d'un coefficient de redistribution, également fixé par la firme et noté $\gamma$. Par construction ce coefficient est compris entre 0 et 1 . Le système est légal dès lors qu'il s'applique de la même manière à tous les actionnaires sans exception ni distinction. Le paramétrage du système est à la discrétion des dirigeants de la firme.

Les actionnaires ne sont pas concernés par la limitation de leur droit de vote si le nombre de titres qu'ils détiennent est en dessous du seuil $\delta$. Nous supposons que c'est le cas de tous les actionnaires passifs et fragmentés. Nous supposerons que cela peut aussi être le cas de certains membres du noyau dur et nous notons B le nombre total de titres détenus par les actionnaires du noyau dur dans cette situation. Compte tenu de la définition de $\mathrm{A}$, l'inégalité $\mathrm{B} \leq \mathrm{A}$ est toujours vérifiée. Inversement, les actionnaires sont concernés par le système de défense s'ils possèdent au moins $\delta$ titres. C'est le cas des autres membres du noyau dur, dont le nombre, nécessairement compris entre 0 and $\mathrm{N}$, sera noté $\mathrm{K}$ dans la suite. Nous supposerons que c'est également toujours le cas de l'investisseur hostile, $h$. Après la mise en place du

\footnotetext{
${ }^{5}$ Sur le plan théorique, les OPA donnent la possibilité aux petits porteurs de réaliser des plus-values en cédant au prix fort leurs actions à un investisseur potentiel. Dans la pratique, on observe que dans un cadre où la vente d'actions reste très encadrée par le législateur (Bagnoli et Lipman [1988]; Bessière [1997]; Grossman et Hart [1980b]), ils n'ont pas intérêt à vendre trop vite leurs actions dans l'espoir d'un surenchérissement (Grossman et Hart [1980a]; Holmstrom et Nalebuff [1992]). Les actionnaires passifs sont, en fait, partagés en deux groupes: les premiers souhaitent vendre immédiatement leurs actions au plus offrant; les second sont attentistes, s'interrogeant sur l'opportunité de différer la vente. Un actionnaire passif n'est, en réalité, vendeur que s'il estime le prix proposé lors de l'OPA supérieur à la valeur future de son action et s'il considère la probabilité d'une surenchère très faible.

${ }^{6}$ En conséquence la probabilité d'occurrence de l'OPA est égale à 1.

${ }^{7}$ En vertu du principe one share one vote.
} 
dispositif, le nombre total de droits de vote détenus par un actionnaire $j$ lors des assemblées générales, est noté $\mathrm{DV}_{j}$. Il se compose de ses droits non limités et des droits limités puis redistribués dont il bénéficie et qui seront noté $\mathrm{P}_{j}$ dans la suite.

$$
\left\{\begin{array}{l}
\text { si } l_{j} \leq \delta \text { alors } \mathrm{P}_{j}=0 \text { et } \mathrm{DV}_{j}=l_{j} \\
\text { si } l_{j}>\delta \text { alors } \mathrm{P}_{j}=\gamma\left(l_{j}-\delta\right) \text { et } \mathrm{DV}_{j}=\delta+\mathrm{P}_{j}=\gamma l_{j}+(1-\gamma) \delta
\end{array}\right.
$$

Si au moins un actionnaire détient un nombre de titres strictement supérieur au seuil $\delta$ alors le nombre total de droits de vote susceptibles d'être exprimés lors des assemblées générales est strictement inférieur au nombre de titres détenus par les actionnaires présents ${ }^{8}$. Ce nombre est noté $\mathrm{C}$, et il s'obtient par addition du nombre de titres non limités et du nombre de droits de vote redistribués. Selon l'annexe A :

$$
\mathrm{C}=\gamma\left(l_{h}+\mathrm{A}\right)+(1-\gamma)(\delta+\delta \mathrm{K}+\mathrm{B})
$$

Le noyau dur détient initialement le contrôle de la firme et souhaite le garder sans partage ${ }^{9}$. L'évolution du pouvoir au sein de la firme est donc un des éléments centraux du modèle et la principale variable qui permet de le mesurer est le pourcentage de droits de vote détenu par les différents actionnaires lors des assemblées générales. Dans la suite, nous nommerons $\% \mathrm{DV}_{j}$ ce pourcentage relativement à l'actionnaire $j$. L'efficacité du système de défense mis en place peut être mesuré par le pourcentage de droits de vote que l'investisseur hostile parvient finalement à détenir, soit :

$$
\% \mathrm{DV}_{h}=\frac{\mathrm{DV}_{h}}{\mathrm{C}}=\frac{\gamma l_{h}+(1-\gamma) \delta}{\gamma\left(l_{h}+\mathrm{A}\right)+(1-\gamma)(\delta+\delta \mathrm{K}+\mathrm{B})}
$$

Avec la mise en place du dispositif de défense, les actionnaires du noyau dur souhaitent limiter le poids de l'investisseur hostile dans le capital de la firme. Dans la suite de l'article, $\mathrm{S}$ désigne le pourcentage maximum des droits de vote que le noyau dur autorise l'investisseur hostile à détenir.

La chronologie des événements peut être résumée de la manière suivante : au temps $t_{0}$ le noyau dur estime la valeur la plus probable du nombre de titres que l'investisseur hostile est susceptible de détenir. Au temps $t_{1}$ le noyau dur fixe les valeurs des paramètres $\delta$ et $\gamma$, et par conséquent celle du seuil $S$, qui semblent constituer le meilleur système de défense contre les OPA. Au temps $t_{2}$ l'investisseur hostile révise sa position sur la base des valeurs de $\delta$, de $\gamma$ et donc de $S$, anticipées par lui. Au temps $t_{3}, \ldots, t_{\mathrm{M}-1}$ le noyau dur et l'investisseur hostile révisent leurs positions respectives en fonction de leurs anticipations. Dans les faits, le noyau dur va modifier les paramètres du système et l'investisseur hostile va réviser à la hausse ou à la baisse le nombre de titres qu'il souhaite détenir à la fin de l'opération. Au

\footnotetext{
${ }^{8}$ Cela est systématiquement le cas à la fin du ramassage des titres car l'investisseur hostile est toujours supposé dépasser ce seuil.

${ }^{9}$ La discussion serait symétrique si on souhaitait se placer du point de vue de l'initiateur de l'OPA.
} 
temps $t_{\mathrm{M}}$, qui représente la fin de la période d'OPA, l'investisseur hostile détient $l_{h}$ titres qui lui octroient $\% \mathrm{DV}_{h}$ droits de vote lors des assemblées générales et les paramètres $\delta, \gamma$ et $\mathrm{S}$ n'évoluent plus.

\section{LA MISE EN EVIDENCE DU SEUIL DEFENSIF}

En théorie $S$ est compris entre 0 et 1 . Pour simplifier le cadre de la modélisation, nous supposerons toujours que $0 \leq S<1$. Cela ne modifie en rien la portée des résultats dans la mesure où le dispositif prend tout son intérêt pour les valeurs de $S$ inférieures à $\frac{1}{2}$. Puisque $h$ agit seul, cet objectif est systématiquement réalisable. Pour cela, il suffit de poser :

$$
\delta<\text { A et } \gamma=0
$$

Un tel choix rend le poids de $h$ systématiquement plus faible que celui du noyau dur historique, ce dernier restant coalisé. Le dispositif est donc toujours capable de contrer un unique investisseur hostile. En pratique, plus la valeur de $\mathrm{S}$ est faible, plus le noyau dur est protégé. Deux valeurs sont particulièrement sensibles, soient $\mathrm{S}=\frac{1}{2}$ et $\mathrm{S}=\frac{1}{3}$ qui correspondent respectivement à la majorité lors des assemblées générales ordinaires et à la minorité de blocage. Le noyau dur peut aussi décider de fixer une valeur de $\mathrm{S}$ inférieure à $\frac{1}{3}$. Cependant, plus le dispositif est contraignant, donc efficace dans son action à limiter le pouvoir de $h$, plus son impact en termes d'image vers le marché est négatif.

La discussion porte désormais sur les valeurs du seuil de limitation et du coefficient de redistribution. Pour contrer efficacement l'investisseur hostile, il faut que la valeur du seuil $\delta$ soit la plus petite possible et que la valeur de $\gamma$ associée soit la plus proche possible de 0 . Cependant, si l'entreprise souhaite garder la confiance des actionnaires minoritaires et continuer à attirer efficacement les investisseurs non hostiles, il faut que le dispositif soit le plus léger possible. En effet, une activation trop violente du système risque de susciter la défiance des petits actionnaires puisque le processus de neutralisation des droits de vote est perçu comme néfaste à la bonne gouvernance. Cela implique donc, cette fois, que la valeur du seuil $\delta$ soit la plus grande possible et que la valeur de $\gamma$ associée, particulièrement observée par le marché, soit la plus proche possible de 1 . Le choix du couple $(\delta, \gamma)$ est stratégique car il définit à la fois l'efficacité du dispositif, son impact en termes d'image négative et le quorum, c'est-à-dire, le pourcentage de droits de vote devant être présents ou représentés lors d'une assemblée générale.

Les situations à la limite sont irréalistes ou sans intérêt. Celles où les valeurs de $\delta$ ou $\gamma$ seraient très faibles ou nulles envoient un signal rédhibitoire au marché en sclérosant tout contrôle possible de l'entreprise. Celles où la valeur de $\delta$ serait très proche de $\mathrm{T}$ ou encore celles où la valeur de $\gamma$ serait très proche de 1 annulent l'intérêt du dispositif puisque la situation serait alors la même qu'en son absence. Logiquement, nous allons nous éloigner de ces cas. Comme nous avons supposé que 
$h$ détient un nombre de titres supérieur à $\delta$, nous considérerons, en particulier, toujours vérifiée la double inégalité :

$$
l_{\mathrm{N}} \leq \delta \leq l_{h}
$$

Nous savons qu'en fonction du prix de l'offre et du profil des actionnaires passifs, les membres du noyau dur sont capables d'estimer le nombre de titres que l'investisseur hostile est susceptible de ramasser. Ce nombre détermine les droits de vote que ce même investisseur détient. Tous les autres paramètres du problème sont connus ou fixés par le noyau dur. D'après l'annexe $\mathrm{B}$, la discussion de l'inégalité $\% \mathrm{DV}_{h}<\mathrm{S}$ conduit à $l_{h}<\sigma_{\mathrm{S}}$, où $\sigma_{\mathrm{S}}$ est défini par :

$$
\sigma_{\mathrm{S}}=\left(\frac{\mathrm{S}}{1-\mathrm{S}}\right)\left(\mathrm{A}+\left(\frac{1-\gamma}{\gamma}\right)\left(\frac{\delta(\mathrm{S}-1)}{\mathrm{S}}+\delta \mathrm{K}+\mathrm{B}\right)\right)
$$

Il apparait donc que le succès de l'OPA dépend de la capacité de l'investisseur hostile à ramasser un nombre de titres supérieur ou égal à $\sigma_{\mathrm{S}}$. Réciproquement, l'efficacité du dispositif mis en place par le noyau dur dépend de sa capacité à maintenir $l_{h}$ en dessous du même seuil $\sigma_{\mathrm{S}}$ que nous nommerons le seuil défensif dans la suite ${ }^{10}$.

\section{L'OPTIMISATION DU PARAMETRAGE DU DISPOSITIF}

Du point de vue de l'efficacité du système, les valeurs de $\delta$ et de $\gamma$, doivent engendrer un seuil défensif qui garantit l'inégalité $l_{h}<\sigma_{\mathrm{S}}$. Du point de vue de l'image renvoyée au marché, les actionnaires du noyau dur doivent, à l'inverse, fixer des valeurs de $\delta$ et de $\gamma$ qui minimisent la valeur du seuil $\sigma_{\mathrm{S}}$, ce dernier étant perçu d'autant plus négativement par le marché qu'il est contraignant. Il est naturellement possible d'associer une fonction de coût à la valeur du seuil défensif $\sigma_{\mathrm{S}}$ mais, dans cet article, nous supposerons simplement que cette fonction est linéaire et croissante avec la valeur de $\sigma_{\mathrm{S}}$. Finalement, les valeurs de $\delta$ et $\gamma$ doivent idéalement définir la plus petite valeur du seuil défensif qui vérifie $l_{h}<\sigma_{\mathrm{S}}$ en fonction des anticipations

\footnotetext{
${ }^{10}$ Nous supposons que le prix offert par $h$ est suffisamment attractif et que l'investisseur hostile n'a pas de difficulté à ramasser le nombre de titres qu'il souhaite, dès lors que ces mêmes titres sont effectivement disponibles sur le marché. Le nombre maximum de titres que $h$ peut théoriquement acheter est égal au nombre total de titres en circulation diminué du nombre de titres détenus par les actionnaires du noyau dur, soit T-A. De fait, l'OPA ne sera effectivement lancée que si le seuil défensif est lui-même inférieur à cette dernière valeur, soit :

$$
\mathrm{A}<(1-\mathrm{S})\left(\mathrm{T}-\left(\frac{\mathrm{S}}{1-\mathrm{S}}\right)\left(\frac{1-\gamma}{\gamma}\right)\left(\frac{\delta(\mathrm{S}-1)}{\mathrm{S}}+\delta \mathrm{K}+\mathrm{B}\right)\right)
$$

Si cette condition n'est pas remplie, toute tentative de prise de contrôle de la firme est vouée à l'échec et les dispositifs de défense deviennent sans objet. C'est la raison pour laquelle, nous la supposerons toujours vraie.
} 
des actionnaires du noyau dur. Comme nous avons postulé que la structure de l'actionnariat est figée à l'intérieur du noyau dur, les paramètres d'action du seuil défensif sont donc réduits au quadruplet $(\delta, \gamma, \mathrm{K}, \mathrm{B})$. Nous devons donc résoudre le programme :

$$
\begin{gathered}
\min _{\delta, \gamma, \mathrm{K}, \mathrm{B}} \\
\text { s. c. } \\
l_{\mathrm{N}} \leq \delta \leq l_{h}, 0 \leq \gamma \leq 1, l_{h}<\sigma_{\mathrm{S}}
\end{gathered}
$$

La matrice hessienne, constituée des quatre dérivées du second ordre de $\sigma_{\mathrm{S}}$ par rapport aux variables $\delta$ et $\gamma$ est décrite dans l'annexe C. La matrice n'étant pas définie positive au sens mathématique du terme ${ }^{11}$, le programme de minimisation ne peut se limiter à l'étude des seules valeurs d'annulation du gradient ${ }^{12}$. Il nous faut donc successivement étudier l'influence de la variation du seuil de limitation et du coefficient de redistribution sur le seuil défensif.

Si on suppose que la valeur initiale de $\delta$ est suffisamment grande pour qu'aucun membre du noyau dur ne soit concerné par la limitation des droits de vote, alors $\delta>l_{1}$ et $\mathrm{K}=0$. L'abaissement progressif de la valeur de $\delta$ va augmenter la valeur de $\mathrm{K}$ par sauts successifs. Ceci aura pour effet d'abaisser la valeur de B, également par sauts. Les paramètres $\delta, \mathrm{K}$ et $\mathrm{B}$ sont donc liés entre eux. En conséquence, pour étudier la sensibilité du seuil $\sigma_{\mathrm{S}}$ à ces trois paramètres, il suffit de l'étudier par rapport au seul paramètre $\delta$. Nous effectuerons, ensuite, ce même travail par rapport au paramètre $\gamma$.

\section{L’influence du seuil de limitation sur le seuil défensif}

Cette étude repose sur l'examen de la dérivée partielle du seuil défensif par rapport à $\delta$ :

$$
\frac{\partial \sigma_{S}}{\partial \delta}=\left(\frac{1-\gamma}{\gamma}\right)\left(\frac{K S}{1-S}-1\right)
$$

Compte tenu des hypothèses émises sur $\mathrm{S}$ et $\gamma$, la dérivée partielle s'annule pour :

$$
\mathrm{S}_{1}=\frac{1}{\mathrm{~K}+1}
$$

La dérivée est positive pour $\mathrm{S}>\mathrm{S}_{1}$ et négative sinon. En conséquence, la décroissance $\delta$ augmente la valeur de $\sigma_{\mathrm{S}}$ tant que $\mathrm{S}<\mathrm{S}_{1}$, elle diminue la valeur de

\footnotetext{
${ }^{11}$ En particulier parce que son déterminant n'est pas systématiquement négatif.

${ }^{12}$ Le gradient est le vecteur constitué des dérivées partielles du premier ordre.
} 
$\sigma_{\mathrm{S}}$ dès que $\mathrm{S}>\mathrm{S}_{1}$ et elle sera sans effet sur la valeur de $\sigma_{\mathrm{S}}$ si $\mathrm{S}=\mathrm{S}_{1}$. Nous savons que la décroissance de $\delta$ augmente, par palier, la valeur de $\mathrm{K}$ et donc diminue, également par palier, celle de $\mathrm{S}_{1}$. Comme nous avons supposé au début du processus de réglage que la valeur de $\delta$ est supérieure à $l_{1}$, la valeur initiale de $\mathrm{K}$ est donc nulle. Dans ce cas premier cas de figure, nous avons $S_{1}=1$ et comme nous avons également supposé $0 \leq \mathrm{S}<1$ alors $\mathrm{S}<\mathrm{S}_{1}$. Dès lors, toute diminution de $\delta$ va, indépendamment de la valeur de $S$, augmenter celle de $\sigma_{S}$. L'intérêt du noyau dur est donc de favoriser un abaissement de $\delta$. Cette action est conduite jusqu'à atteindre la valeur $\delta=l_{1}$ qui va engendrer le changement de la valeur de $\mathrm{K}$, avec $K=1$. Dans ce deuxième cas de figure, la nouvelle valeur de $S_{1}$ est $S_{1}=\frac{1}{2}$.

Désormais, la démarche n'est plus indépendante de $\mathrm{S}$. Deux cas doivent être distingués : $\left(S_{1}=\frac{1}{2}\right.$ avec $\left.S=\frac{1}{2}\right)$ et $\left(S_{1}=\frac{1}{2}\right.$ avec $\left.S<\frac{1}{2}\right)$. Si l'objectif du noyau dur est d'assurer une valeur de $\mathrm{S}$ exactement égale à $\frac{1}{2}$, alors $\mathrm{S}=\mathrm{S}_{1}$. Nous savons que dans ce cas, les modifications de la valeur de $\delta$ sont sans effet sur $\sigma_{\mathrm{S}}$. il n'est plus nécessaire de modifier la valeur de $\delta$. En effet, une nouvelle décroissance de $\delta$ jusqu'à la valeur $\delta=l_{2}$, sera sans effet sur la valeur de $\sigma_{\mathrm{S}}$ mais aura des conséquences négatives en termes d'image. Une décroissance de $\delta$ en dessous de $l_{2}$ augmente la valeur de $K$ avec $K=2$ et donc abaisse celle de $S_{1}$, avec $S_{1}=\frac{1}{3}$. Avec cette nouvelle valeur de $S_{1}$, la variable $S$ devient strictement supérieure à $S_{1}$, ce qui engendre une dégradation de la valeur de $\sigma_{S}$, ce qui n'est pas souhaitable. C'est donc bien $\delta=l_{1}$ qui assure le meilleur réglage.

Finalement, si $\frac{1}{2} \leq \mathrm{S}<1, \delta=l_{1}$ est le meilleur réglage pour $\delta$.

Considérons maintenant la situation $\left(\mathrm{S}_{1}=\frac{1}{2}\right.$ avec $\left.\mathrm{S}<\frac{1}{2}\right)$. On suppose que la valeur de $\mathrm{S}$ est supérieure ou égale à $\frac{1}{3}$. Nous sommes dans le cas où $\mathrm{S}<\mathrm{S}_{1}$. Nous savons que dans ce cas, la décroissance de $\delta$ entraine un accroissement de $\sigma_{\mathrm{S}}$. Si l'objectif du noyau dur est, en réalité, d'assurer une valeur de $\mathrm{S}$ strictement inférieure à $\frac{1}{2}$ (mais strictement supérieure à $\frac{1}{3}$ ) le réglage $\delta=l_{1}$ n'est plus valable et il convient de diminuer $\delta$. Tant que sa valeur reste strictement supérieure à $l_{2}$, l'abaissement de $\delta$ se traduit par une augmentation de la valeur de $\sigma_{\mathrm{S}}$. Dès que $\delta$ devient strictement inférieur à $l_{2}$, l'augmentation de la valeur de $\mathrm{K}$ devient effective avec $\mathrm{K}=2$. Dans ce troisième cas de figure, nous avons $\mathrm{S}_{1}=\frac{1}{3}$.

Deux nouveaux cas doivent être distingués : $\left(S_{1}=\frac{1}{3}\right.$ avec $\left.S=\frac{1}{3}\right)$ et $\left(S_{1}=\frac{1}{3}\right.$ avec $\mathrm{S}<\frac{1}{3}$ ). Si l'objectif du noyau dur est d'assurer une valeur de $\mathrm{S}$ exactement égale à 
$\frac{1}{3}$, nous sommes de nouveau dans la situation $S=S_{1}$ et les changements de la valeur de $\delta$ sont sans effet sur $\sigma_{\mathrm{s}}$. Il n'est donc plus nécessaire de modifier la valeur de $\delta$. En réalité, une nouvelle décroissance de la valeur de $\delta$, jusqu'à la valeur $\delta=l_{3}$ ne modifie pas la valeur de $\sigma_{\mathrm{S}}$ mais, comme dans le cas précèdent, aura un impact négatif sur le marché en termes d'image. Une décroissance de $\delta$ sous la valeur de $l_{3}$ va augmenter la valeur de $K$ avec $K=3$, ce qui va diminuer la valeur de $S_{1}$, avec $\mathrm{S}_{1}=\frac{1}{4}$. Avec cette nouvelle valeur de $\mathrm{S}_{1}$, la variable $\mathrm{S}$, qui est toujours égale à $\frac{1}{3}$, devient strictement supérieure à $S_{1}$. Cela implique une diminution de la valeur de $\sigma_{\mathrm{S}}$, ce qui, là encore, n'est pas souhaitable. Dans le cas de figure $\left(S_{1}=\frac{1}{3}\right.$ avec $\mathrm{S}=\frac{1}{3}$ ), c'est donc bien $\delta=l_{2}$ qui assure le meilleur réglage.

Finalement, si $\frac{1}{3} \leq \mathrm{S}<\frac{1}{2}, \delta=l_{2}$ est le meilleur réglage de $\delta$.

Le cas $\left(S_{1}=\frac{1}{3}\right.$ avec $\left.\mathrm{S}<\frac{1}{3}\right)$ et les suivants sont traités dans l'annexe D qui propose une démonstration par récurrence de la proposition (10) et qui généralise les résultats obtenus aux rangs 1 et 2 . Finalement, si on note $\delta_{0}$ le meilleur réglage ${ }^{13}$ pour $\delta$ :

$$
\text { si } \frac{1}{k+1} \leq \mathrm{S}<\frac{1}{k} \text {, alors } \delta_{0}=l_{k}
$$

A ce stade, il apparait que la composition du noyau dur n'est pas sans importance. Le nombre $\mathrm{N}$ d'actionnaires de ce noyau détermine la limite que l'on peut atteindre pour $S$, soit $S=\frac{1}{N+1}$. Par ailleurs, puisque la finesse de réglage du dispositif repose sur les différentes valeurs des $l_{j}$, il est préférable qu'aucun membre du noyau dur ne possède le même nombre de titres.

\section{L'influence du coefficient de redistribution sur le seuil défensif}

Cette fois, il nous faut étudier le comportement de la dérivée partielle du seuil défensif par rapport à $\gamma$ :

$$
\frac{\partial \sigma_{S}}{\partial \gamma}=\left(\frac{-1}{\gamma^{2}}\right)\left(\frac{S}{1-S}\right)\left(\frac{\delta(S-1)}{S}+\delta K+B\right)
$$

Compte tenu des hypothèses sur $\mathrm{S}$ et sur $\gamma$ la dérivée partielle s'annule pour :

\footnotetext{
${ }^{13}$ On rappelle que par hypothèse $\mathrm{K}$ est un entier compris entre 0 et $\mathrm{N}$. Comme la situation $\mathrm{K}=0$ est par construction écartée, nécessairement, $k$ est également un nombre entier et $k>0$.
} 


$$
\mathrm{S}_{2}=\frac{1}{\mathrm{~K}+1+\frac{\mathrm{B}}{\delta}}
$$

Elle est négative si $\mathrm{S}<\mathrm{S}_{2}$ et positive si $\mathrm{S}>\mathrm{S}_{2}$. Or, on observe que la valeur de $\mathrm{S}_{2}$ est toujours strictement inférieure à celle de $S_{1}$ et donc pour tout $k$ entier non nul :

$$
\begin{gathered}
\text { si } \frac{1}{k+1} \leq \mathrm{S}<\frac{1}{k}, \text { alors } \mathrm{S}_{2}=\frac{1}{k+1+\frac{\mathrm{B}}{\delta}}<\mathrm{S}_{1}=\frac{1}{k+1} \leq \mathrm{S} \\
\text { et donc } \frac{\partial \sigma_{\mathrm{S}}}{\partial \gamma}<0
\end{gathered}
$$

Du point de vue de la stricte efficacité numérique, les actionnaires du noyau dur doivent donc théoriquement et fort logiquement choisir la plus petite valeur de $\gamma$. Cependant, l'impact sur le marché du coefficient de redistribution est supposé très important, ce qui conduit au choix inverse d'une valeur de $\gamma$ la plus élevée possible. La valeur de $\delta$ étant maintenant fixé à $\delta_{0}$ pour préserver l'attractivité de l'entreprise, le noyau dur va donc choisir la plus grande valeur de $\gamma$ qui vérifie $l_{h}<\sigma_{\mathrm{S}}$. Les calculs de l'annexe E conduisent à $\gamma<\gamma_{0}$ avec :

$$
\gamma_{0}=\frac{\delta_{0}(\mathrm{~S}(1+k)-1)+\mathrm{SB}}{l_{h}(1-\mathrm{S})-\mathrm{SA}+\delta_{0}(\mathrm{~S}(1+k)-1)+\mathrm{SB}}
$$

\section{Cas particuliers et limites}

Comme $\mathrm{S}$ est supérieur ou égal à $\frac{1}{k+1}$, le numérateur de l'expression de $\gamma_{0}$ est toujours positif. Il en est de même pour le dénominateur en raison de l'hypothèse $l_{h}>\mathrm{A}$. Dès lors, la valeur de $\gamma_{0}$ est toujours définie et le dispositif ne connaît pas de limite d'application. Fort logiquement, une faible valeur de $l_{h}$ associée à une forte valeur de A renvoient des valeurs de $\gamma_{0}$ proches de 1 , ce qui confirme que la possession d'un grand nombre de titres par le noyau dur constitue la première étape d'une bonne protection anti-OPA. Dans le cas contraire, la valeur de $\gamma_{0}$ pourra rapidement se dégrader et tendre vers 0 , au détriment du signal envoyé au marché.

Deux situations sont particulièrement observées. La première correspond au verrouillage de la majorité des droits de vote, soit $S=\frac{1}{2}$; la seconde au verrouillage de la minorité de blocage, soit $S=\frac{1}{3}$. L'application des résultats précédents à ces deux cas particuliers nous donne en termes de réglage du dispositif, respectivement : 


$$
\left\{\begin{array}{l}
\mathrm{K}=1, \delta_{0}=l_{1}, \mathrm{~B}=\mathrm{A}-l_{1} \text { et } \gamma_{0}=\frac{\mathrm{A}-l_{1}}{l_{h}-l_{1}} \\
\mathrm{~K}=2, \delta_{0}=l_{2}, \mathrm{~B}=\mathrm{A}-l_{1}-l_{2} \text { et } \gamma_{0}=\frac{\mathrm{A}-l_{1}-l_{2}}{2 l_{h}-l_{1}-l_{2}}
\end{array}\right.
$$

\section{LA PRISE EN COMPTE DU QUORUM}

\section{L'introduction de la contrainte de quorum}

Les valeurs des paramètres du dispositif vont aussi déterminer le quorum, c'est-àdire le rapport du nombre de droits de vote présents ou représentés en assemblée sur le nombre total de droits de vote. En l'absence du dispositif de limitation des droits de vote, le quorum est donné par le ratio:

$$
\left\{\begin{array}{l}
\frac{\mathrm{A}}{\mathrm{T}} \text { avant l'arrivée de l'investisseur hostile } \\
\frac{l_{h}+\mathrm{A}}{\mathrm{T}} \text { après son arrivée }
\end{array}\right.
$$

Notons que le quorum est calculé sur la base du nombre de droits de vote et non sur celle du nombre total de titres émis. Tout changement du nombre de droits de vote aura un impact sur le quorum. Le choix de ces valeurs ne doit pas entraver le fonctionnement normal de l'entreprise et il est primordial de préserver le quorum minimum exigé pour la tenue des assemblées générales afin d'éviter les convocations à répétition et les retards dans les décisions d'ordre stratégique. On note $\frac{1}{q}$ le quorum minimum ${ }^{14}$ que les actionnaires du noyau dur souhaitent préserver. On suppose que ce quorum minimum est atteint avant la mise en place du dispositif, ce qui induit que les actionnaires du noyau dur disposent d'un nombre de titres suffisants. On note Q le quorum atteint après la mise en place du dispositif. Le problème initial est donc augmenté d'une contrainte supplémentaire. Nous devons maintenant résoudre le nouveau programme :

$$
\begin{gathered}
\min _{\delta, \gamma, \mathrm{K}, \mathrm{B}} \sigma_{\mathrm{S}} \\
\text { s. c. } \\
l_{\mathrm{N}} \leq \delta \leq l_{h}, 0 \leq \gamma \leq 1, l_{h}<\sigma_{\mathrm{S}}, \quad \frac{1}{q} \leq \mathrm{Q}
\end{gathered}
$$

En toute logique, après avoir ramassé ses titres et même s'il ne parvient pas à détenir la majorité des droits de vote, l'investisseur hostile va participer aux assemblées pour faire entendre son point de vue. Dans ce premier cas de figure, si $\mathrm{Q}_{h+}$ est le quorum après activation du dispositif de limitation, alors $\mathrm{Q}=\mathrm{Q}_{h_{+}}$avec :

\footnotetext{
${ }^{14}$ En principe, le quorum minimum dépend, de la nature juridique de la société, du caractère ordinaire ou extraordinaire de l'assemblée et du rang de la convocation. Il est souvent renforcé statutairement par rapport aux exigences du législateur.
} 


$$
\mathrm{Q}_{h+}=\frac{\mathrm{C}}{\mathrm{T}-\left(l_{h}+\mathrm{A}\right)+\mathrm{C}}
$$

Dans cette expression, le numérateur est le nombre de droits de vote présents ou représentés lors des assemblées générales, après limitation. Le dénominateur est le nombre total de titres $\mathrm{T}$ diminué du nombre de titres des actionnaires présents lors des assemblées $l_{h}+\mathrm{A}$ et remplacé par le nombre de droits de vote qui leur sont équivalents après le processus de limitation, soit $\mathrm{C}$.

Nous devons cependant envisager le cas où $h$ décide de ne pas participer aux assemblées, par exemple pour empêcher ou retarder une décision qu'il sait être négative pour lui. Dans ce cas de figure, si $\mathrm{Q}_{h-}$ est le quorum après activation du dispositif de limitation, alors $\mathrm{Q}=\mathrm{Q}_{h-}$ avec :

$$
\mathrm{Q}_{h-}=\frac{\mathrm{C}-\mathrm{DV}_{h}}{\mathrm{~T}-\left(l_{h}+\mathrm{A}\right)+\mathrm{C}}
$$

\section{L'impact de la contrainte de quorum sur le réglage du dispositif}

Numériquement, avec un réglage du dispositif sur la base du couple $\left(\delta_{0}, \gamma_{0}\right)$, on observe que le quorum minimum est généralement atteint sans difficulté tant que l'exigence sur S reste limitée, c'est-à-dire tant que $\mathrm{S}$ est assez proche de $\frac{1}{2}$. Dans ces cas de figure, le paramétrage optimum du dispositif est donc bien constitué du couple $\left(\delta_{0}, \gamma_{0}\right)$. Si le noyau dur recherche de plus faibles valeurs de $S$, le réglage obtenu sur la même base peut ne plus respecter le quorum minimum. La nouvelle contrainte $\frac{1}{q} \leq \mathrm{Q}$ impose alors d'augmenter la valeur de $\delta$ pour diminuer l'effet de la limitation des droits de vote. Cette décision dégrade nécessairement le réglage puisque, $\mathrm{S}$ étant fixé, toute augmentation de $\delta$ s'accompagne d'une diminution de $\gamma$ néfaste en termes d'image. L'annexe $\mathrm{F}$ donne, à partir du réglage initial $\left(\delta_{0}, \gamma_{0}\right)$ la plus petite valeur que $\delta$ doit théoriquement atteindre pour que la condition de quorum soit respectée. Les valeurs de $r, s, t, u, v$ et $w$ étant également données en annexe F, on obtient :

$$
\left\{\begin{array}{l}
\delta=\delta_{1}^{h+}=\frac{r \mathrm{~B}+s}{-r \mathrm{~K}+t} \text { si } h \text { participe aux assemblées } \\
\delta=\delta_{1}^{h-}=\frac{u \mathrm{~B}+v}{-u \mathrm{~K}+w} \text { sinon }
\end{array}\right.
$$

Concentrons-nous dans un premier temps sur la valeur de $\delta_{1}^{h+}$. Son expression complète dépend de l'ensemble des variables d'action du dispositif, soit $\mathrm{K}$ et $\mathrm{B}$ (qui évoluent en fonction du niveau de $\delta$ ) puis $\mathrm{S}, \mathrm{T}, q$, A et $l_{h}$ (que l'on retrouve dans les expressions de $r, s, t, u, v$ et $w$ qui sont indépendantes de $\delta$ ). Ces dernières 
valeurs sont connues, fixées ou anticipées par les actionnaires du noyau dur. La valeur de $\delta_{1}^{h+}$ est donc calculée sur la base des valeurs de $\mathrm{K}=k$ et de $\mathrm{B}=\mathrm{A}-l_{1}-\ldots-l_{k}$ qui correspondent à l'optimum, hors contrainte de quorum, soit $\delta_{0}=l_{k}$. On peut donc écrire :

$$
\delta_{1}^{h+}=\frac{r\left(\mathrm{~A}-l_{1}-\ldots-l_{k}\right)+s}{-r k+t}
$$

Le calcul de $\delta_{1}^{h+}$ n'est utile que si le quorum n'est pas atteint avec le paramétrage $\left(\delta_{0}, \gamma_{0}\right)$ et nécessairement $\delta_{1}^{h+}>\delta_{0}=l_{k}$. La recherche du dépassement du quorum minimum impose d'abandonner le premier choix $\delta=\delta_{0}$ au profit du deuxième choix $\delta=\delta_{1}^{h+}$ qui implique le franchissement du seuil $l_{k}$ par le paramètre $\delta$. Dès lors, $\mathrm{K}$ et $\mathrm{B}$ deviennent, respectivement, supérieur ou égal à $k-1$ et à $\mathrm{A}-l_{1}-\ldots-l_{k-1}$. On ne change pas les données du problème en posant désormais $\mathrm{K}=k-1$ et $\mathrm{B}=\mathrm{A}-l_{1}-\ldots-l_{k-1}$. Le calcul de la plus petite valeur de $\delta$ pour que le quorum minimum soit atteint se trouve modifié et on obtient :

$$
\delta_{2}^{h+}=\frac{r\left(\mathrm{~A}-l_{1}-\ldots-l_{k-1}\right)+s}{-r(k-1)+t}>\delta_{1}^{h+}
$$

La recherche du dépassement du quorum minimum impose alors d'abandonner le deuxième choix $\delta=\delta_{1}^{h+}$ au profit d'un troisième choix $\delta=\delta_{2}^{h+}$ qui, cette fois, n'implique pas systématiquement le franchissement du seuil $l_{k-1}$ par le paramètre $\delta$. Si le franchissement a lieu il faut recommencer l'opération précédente qui conduit au calcul de $\delta_{3}^{h+}$ avec $\delta_{3}^{h+}>\delta_{2}^{h+}$. Dans le cas contraire les paramètres $\mathrm{K}$ et $\mathrm{B}$ restent, cette fois-ci, fixes et $\delta_{3}^{h+}=\delta_{2}^{h+}$. Cela définit $\delta_{3}^{h+}$ comme un point d'équilibre. Finalement, si on note $\delta_{l}^{h+}$ la limite de la suite $\left(\delta_{j}^{h+}\right)_{j \in \square^{*}}$, si on désigne par $\left(\delta^{*}, \gamma^{*}\right)$ le paramétrage optimal du dispositif et si on appelle $\left(\mathrm{K}^{*}, \mathrm{~B}^{*}\right)$ les valeurs de $\mathrm{K}$ et de $\mathrm{B}$ qui correspondent à la valeur de $\delta^{*}$ alors, cette fois, sous condition de respect du quorum minimum :

$$
\delta^{*}=\max \left(\delta_{0}, \delta_{l}^{h+}\right) \text { et } \gamma^{*}=\frac{\delta^{*}\left(\mathrm{~S}\left(1+\mathrm{K}^{*}\right)-1\right)+\mathrm{SB}^{*}}{\delta^{*}\left(\mathrm{~S}\left(1+\mathrm{K}^{*}\right)-1\right)+\mathrm{SB}^{*}+l_{h}(1-\mathrm{S})-\mathrm{SA}}
$$

Le raisonnement est en tout point identique avec la suite $\left(\delta_{j}^{h-}\right)_{j \in \square^{*}}$ et le positionnement sur l'une ou l'autre suite dépendant exclusivement du choix stratégique du noyau dur.

\section{Cas particuliers et limites}

Si la contrainte de quorum est ignorée, nous savons que le dispositif est 
systématiquement efficace, en ce sens qu'il existe toujours un paramétrage capable de mettre en échec l'attaque d'un investisseur hostile unique. Sur les nombreuses simulations numériques réalisées à partir de notre modélisation, nous observons que cela n'est plus systématiquement vrai si un quorum minimum est imposé. Fort naturellement, le dispositif perd de son efficacité avec des valeurs de $\mathrm{S}$ et de $q$ faibles, c'est-à-dire si on impose à la fois un pourcentage de droits de vote maximum pour $h$ faible et un quorum minimum élevé. En effet, une telle situation va engendrer des valeurs importantes pour $\delta^{*}$ et faibles pour $\mathrm{K}^{*}$; si la valeur de $\mathrm{S}$ est elle-même faible, les signes de $\mathrm{S}\left(1+\mathrm{K}^{*}\right)-1$ et de $\delta^{*}\left(\mathrm{~S}\left(1+\mathrm{K}^{*}\right)-1\right)+\mathrm{SB}^{*}$ peuvent alors devenir négatifs, ce qui constitue une limite théorique d'application du dispositif. La valeur de $\delta^{*}$ n'étant pas indépendante de $S$ cette limite n'est définie que de manière implicite.

\section{IMPACT DE LA PRISE EN COMPTE DE L'INFORMATION IMPARFAITE SUR LE MODELE}

Dans l'ensemble des précédentes sections, le cadre du modèle est déterministe. L'information est connue et l'occurrence de l'OPA est certaine. Ce cadre est essentiellement théorique. En situation réelle l'apparition d'un ou plusieurs investisseurs hostiles est un phénomène aléatoire. L'étude complète des stratégies de défense reposant sur la limitation des droits de vote en information imparfaite fera l'objet de recherches ultérieures. Nous voulons cependant, dans cette nouvelle section, introduire un premier élément non déterministe et relâcher l'une des hypothèses du cadre initial du modèle. Ainsi, dans la suite, nous postulons désormais que l'investisseur hostile lance son OPA avec une probabilité $p$. En conséquence, il n'engage aucun rachat d'action avec une probabilité $1-p$. Fort logiquement, la probabilité d'occurrence de l'OPA est d'autant plus faible que le système de défense mis en place est dissuasif et la valeur de $p$ dépend du niveau des paramètres $\delta$ et $\gamma: p_{(\delta, \gamma)}$.

L'impact fondamental de la prise en compte de l'information imparfaite sur le modèle initial concerne la valeur de $\% \mathrm{DV}_{h}$ qu'il faut désormais remplacer par la valeur de son espérance $\mathrm{E}\left(\% \mathrm{DV}_{h}\right)$. Si l'investisseur hostile lance l'OPA, compte tenu des hypothèses, le nombre de droits de vote de $h$, la valeur de $\mathrm{DV}_{h}$ et celle de $\mathrm{C}$ restent identiques au cas déterministe avec $\mathrm{DV}_{h}=\gamma l_{h}+(1-\gamma) \delta$ et $C=\gamma\left(l_{h}+\mathrm{A}\right)+(1-\gamma)(\delta+\delta \mathrm{K}+\mathrm{B})$. Si, l'investisseur hostile estime ses chances de contrôle trop faibles, il ne lancera pas son OPA et dans ce cas $\mathrm{DV}_{h}=0$. Dans ces conditions, l'espérance recherchée est donc :

$$
\mathrm{E}\left(\% \mathrm{DV}_{h}\right)=p_{(\delta, \gamma)} \frac{\gamma l_{h}+(1-\gamma) \delta}{\gamma\left(l_{h}+\mathrm{A}\right)+(1-\gamma)(\delta+\delta \mathrm{K}+\mathrm{B})}
$$

Si S désigne toujours le pourcentage maximum des droits de vote que le noyau dur autorise l'investisseur hostile à détenir, selon l'annexe $\mathrm{G}$, la discussion de l'inégalité 
$\mathrm{E}\left(\% \mathrm{DV}_{h}<\mathrm{S}\right)$ conduit désormais à $l_{h}<\sigma_{\mathrm{S}}^{p}$. Sous réserve que $p$ soit différent de $S$, le seuil $\sigma_{\mathrm{S}}^{p}$ est alors défini par :

$$
\sigma_{\mathrm{S}}^{p}=\left(\frac{\mathrm{S}}{p_{(\delta, \gamma)}-\mathrm{S}}\right)\left(\mathrm{A}+\left(\frac{1-\gamma}{\gamma}\right)\left(\frac{\delta\left(\mathrm{S}-p_{(\delta, \gamma)}\right)}{\mathrm{S}}+\delta \mathrm{K}+\mathrm{B}\right)\right)
$$

L'introduction de l'information imparfaite confirme l'existence du seuil défensif mis en évidence dans le cas déterministe. L'expression du nouveau seuil $\sigma_{\mathrm{S}}^{p}$ intègre désormais la probabilité d'arrivée de l'investisseur hostile $p_{(\delta, \gamma)}$. Son expression est plus complexe mais dépend des mêmes variables d'action que dans le cas déterministe. Son optimisation repose désormais sur la connaissance de l'expression de $p$ en fonction des paramètres $\delta$ et $\gamma$. Plusieurs hypothèses peuvent être envisagées à ce sujet et cette étude fera l'objet d'un prochain article. Le problème d'optimisation est similaire au cas déterministe et consiste en la résolution du programme :

$$
\begin{gathered}
\min _{\delta, \gamma, \mathrm{K}, \mathrm{B}}^{p} \\
\text { s. c. } \\
l_{\mathrm{N}} \leq \delta \leq l_{h}, 0 \leq \gamma \leq 1, l_{h}<\sigma_{\mathrm{S}}^{p}
\end{gathered}
$$

Ce nouveau programme d'optimisation permettra d'étudier de manière approfondie un certain nombre de résultats intuitifs. Le comportement de $\sigma_{\mathrm{S}}^{p}$ en fonction de l'évolution de la probabilité $p$, est au cœur des développements futurs. Ce point pourra faire l'objet d'une étude théorique approfondie. Bien évidemment, la recherche de l'optimum sera d'autant plus aisée que l'expression de $p$ en fonction des paramètres $\delta$ et $\gamma$ est simple.

Comme dans le cas déterministe, une nouvelle contrainte, soit $\frac{1}{q} \leq \mathrm{Q}$, doit être ajoutée si l'on souhaite prendre en compte le quorum lors des assemblées générales.

\section{CONCLUSION}

Cet article analyse du point de vue d'une coalition d'actionnaires qui contrôlent une entreprise et dont les intérêts sont parfaitement alignés - le noyau dur - le recours à des restrictions de vote en tant que mécanismes destinés à dissuader la prise de contrôle par un acquéreur hostile. Le choix des membres du noyau dur s'établit dans un cadre où chaque actionnaire peut exercer pleinement ses droits de vote jusqu'à un seuil de limitation noté $\delta$ et où chaque action supplémentaire au-delà de ce seuil contient seulement une fraction de droit de vote, en fonction d'un coefficient de répartition noté $\gamma$. 
Nous montrons que le choix optimal portant sur $\delta$ et $\gamma$ découle de l'optimisation d'une fonction qui dépend fortement de la structure initiale du capital. Lorsque les actionnaires unissent leurs forces contre un seul investisseur hostile, la mesure de protection basée sur la limitation linéaire des droits de vote est très efficace. Ce résultat confirme la thèse de l'enracinement des dirigeants et de leur capacité à garder le pouvoir, même s'ils détiennent une part minoritaire du capital. Par ailleurs, le nombre d'actionnaires alliés, membres du noyau dur de la société cible, et la clef de répartition des titres dont ils disposent ensemble déterminent directement les limites du pouvoir qu'un investisseur hostile peut espérer obtenir lors des assemblées générales.

Les premières parties de l'article se placent dans un cadre déterministe quant au déclenchement de l'OPA. Le choix optimal de $\delta$ et $\gamma$ résulte alors de l'arbitrage entre le désir du noyau dur de se protéger et la perte de confiance qui risque de se produire s'il applique le dispositif de manière trop brutale. Notre étude détermine les valeurs optimales $\left(\delta_{0}, \gamma_{0}\right)$ qui permettent de limiter son impact négatif sur le marché tout en contrant l'attaque de l'investisseur hostile. En ignorant les conditions de quorum (qui assurent que les assemblées générales ne seront pas paralysées), nous montrons qu'une double solution existe dans tous les cas, même si elle se rapproche parfois des valeurs extrêmes. L'étude détermine également les valeurs optimales $\left(\delta^{*}, \gamma^{*}\right)$ qui répondent cette fois aux conditions de quorum. En toute logique, ce nouveau couple de solutions présente des valeurs qui sont inférieures ou égales aux solutions initiales $\left(\delta_{0}, \gamma_{0}\right)$ ce qui rend le dispositif plus agressif aux yeux du marché. Par ailleurs, l'article montre que dans ce cas, les valeurs $\left(\delta^{*}, \gamma^{*}\right)$ ne sont plus systématiquement déterminées. Ce dernier point met en évidence une limite du mécanisme anti-OPA à limitation des droits de vote.

La dernière partie de l'article complète le problème initial en relâchant l'hypothèse déterministe quant au déclenchement de l'OPA. Ce cas, qui retient une hypothèse d'information imparfaite, confirme les principaux résultats du cadre déterministe. Cependant, il montre que l'existence d'un couple de solutions n'est plus systématique et que la recherche de l'optimum, quand il existe, est plus complexe.

Le papier ouvre de nombreuses perspectives pour la recherche. Dans le cadre déterministe, le dispositif de limitation des droits de vote pourrait être testé dans une configuration non linéaire. On peut aussi imaginer le cas d'une action concertée entre plusieurs investisseurs hostiles. On peut encore s'intéresser à la possible défection d'un ou de plusieurs membres de la coalition, conduisant à un affaiblissement du noyau dur initial des actionnaires. Dans le cadre incertain, on peut imaginer différentes hypothèses concernant le lien entre la probabilité d'occurrence de l'OPA et les valeurs du couple $(\delta, \gamma)$. On peut aussi modéliser le comportement des actionnaires du troisième groupe, considérés ici comme passifs, mais qui pourraient être tentés de négocier préalablement leurs droits de vote en vue d'influencer les décisions de l'entreprise.

\section{REMERCIEMENTS}


Les auteurs remercient Nadia Sghaier pour ses remarques et commentaires. Naturellement, les auteurs restent seuls responsables de leurs développements ou interprétations.

\section{RÉFERENCES BIBLIOGRAPHIQUES}

Agrawal, A., MandelKer, G., [1990], «Large Shareholders and Monitoring of Managers: the Case of Anti-takeover Charter Amendments», Journal of Financial and Quantitative Analysis, 25, p. 143-161.

Armstrong, S., LANGe, H.P., WoO, L.E., [1994], «Can Anti-takeover Activity Really Create Wealth», Asia Pacific Journal of Management, 11, p. 327-343.

AussillouX, V., BessiERE, V., [1998], «OPA. Dissuasion ou prix minimal, offre hostile ou négociée», Revue Economique, 49 (3), p. 777-784.

Austen-SMith, D., O'BRIEN, P., [1992], «Takeover Defences and shareholder Voting», Economica 59, p. 199-219.

Bagnoli, M., LiPMAN, B., [1988], «Successful Takeovers without Exclusion», Review of Financial Studies, 1 (1), p. 89-110.

Bagnoli, M., GoRdOn, R., LIPMAN B., [1989], «Stock Repurchase as a Takeover Defense», Review of Financial Studies, 2 (3), p. 423-443.

BARRY, J., HATFIELD, J., [2012], «Pills and Partisans: Understanding Takeover Defenses», University of Pennsylvania Law Review, 160 (3), p. 633-713.

Bebchuk, L., Coates, J., Subramanian, G., [2002], «The Powerful Anti-takeover force of Staggered Boards : Theory, Evidence and Policy», Stanford Law Review, 54, p. 887-951.

Bebchuk, L., Cohen A., [2005], «The Costs of Entrenched Boards», Journal of Financial Economics, 78, p. 409-433.

Bebchuk, L., CoHEN, A., Ferrell, A., [2009], «What Matters in Corporate Governance?», Review of Financial Studies, 22, p. 783-827.

BeN Ali C., [2014]. « Corporate Governance, Principal-Principal Agency conflicts, and Disclosure», Working Papers 2014-125, Department of Research, Ipag Business School.

Ben Ali C., Teulon, F., [2014]. « CEO Monitoring and board effectiveness - Resolving CEO compensation issue», Working Papers 2014-045, Department of Research, Ipag Business School.

BEssiÈRE, V., [1997], «Takeover Bid and Game Theory an Empirical Investigation on the French Market», Bankers, Markets, Investors, 31, p. 28-38.

CoATES, J., [2000], «Takeover Defenses in the Shadow of the Pill: a Critique of the Scientific Evidence », Texas Law Review, 79, p. 271-382.

Comment, R., Schwert, W., [1995], «Poison or Placebo? Evidence on Deterrence and Wealth Effects of Modern Anti-takeover Measures», Journal of Financial Economics, 39, p. 3-43.

Davidson, W., PILGer, T., SzaKmary, A., [2004], «The Importance of Boards Composition and Committee Structure: the Case of Poison Pills», Corporate Ownership and Control, 1, p. 8195.

DeAngelo, H., RiCE, E. M., [1983], «Anti-takeover Charter Amendments and Stockholder Wealth», Journal of Financial Economics, 11, p. 329-360.

Goldman, E., QIAN, J., [2005], «Optimal Toeholds in Takeover Contests», Journal of Financial Economics, 77, p. 321-346.

Grossman, S., Hart, O., [1980a], «Takeover Bids, the Free-rider Problem and the Theory of Corporation», The Bell Journal of Economics, 11, p. 42-64.

Grossman, S., HART, O., [1980b], «Disclosure Laws and Takeover Bids», Journal of Finance, 35, p. 323-334.

Grossman, S., Hart, O., [1988], «One Share One Vote and the Market for Corporate Control», Journal of Financial Economics, 20, p. 175-202.

HARRIS, M., RAVIV, A., [1988], «Corporate Governance: Voting Rights and Majority Rules», Journal of Financial Economics, 20, p. 203-236.

Heron, R. A., LIE, E., [2006], «On the Use of Poison Pills and Defensive Payouts by Takeover Targets», Journal of Business, 79, p. 1783-1807.

HiRschleIFER, D., TITMAN, S., [1998], «Share Tendering Strategies and the Success of Hostile Takeover», Journal of Political Economy, 98, p. 295-324. 
HolmströM, B., NALEBUfF, B., [1992], «To the Raider Goes the Surplus? A reexamination of the Free-rider Problem», Journal of Economics and Management Strategy, 1 (1), p. 37-62.

HwAnG, S., KIM, W., [2011], «Anti-takeover Charter Amendments and Managerial Entrenchment : Evidence from Korea», KDI School of Public Policy Working Paper.

JAREll, G., POUlSEN A., [1987], «Shark Repellents and Stock Prices: the Effect of Anti-takeover Amendments since 1980», Journal of Financial Economics, 19, p. 127-169.

Jensen, M., [1986], «Agency Costs of Free Cash Flow, Corporate Finance and Takeover», American Economic Review, 76, p. 323-329.

Jensen, M., [1988], «Takeovers: Their Causes and Consequences», Journal of Economic Perspective, 2, p. 21-48.

Johnson, M., RAO, P., [1997], «Does Anti-takeover Amendments Reduce Myopic Managerial Investment Behavior ?», Journal of Managerial Issues, 9, p. 497-511.

LA PORTA, R., LOPEZ-DE-SIlANES, F., SHLEIFER, A., VISHNY, R., [1998], «Law and Finance», Journal of Political Economy, 106, p. 1113-1155.

MaletTe, P., Fowler, K., [1993], «Effect of Board Composition and Stock Ownership on the Adoption of Poison Pills», Academy of Management Journal, 35, p.1010-1035.

MoLIN, J., [1996], « Optimal Deterrence and Inducement of takeovers: an analysis of Poison Pills and dilution», in Essays on Corporate Finance and Governance, Stockholm School of Economics, p. 73-104.

Moschetto B.-L., Teulon, F., [2013], «Linear voting rule limitation strategy to reduce the power of a unique new comer in a firm's capital », Working Papers 2013-001, Department of Research, Ipag Business School.

PECK, S., [2004], «MBO Financing Risks and Managers' Use of Anti-takeover Deterrance», The Journal of Applied Business Research, 20 (3), p.11-30.

Rechner, P., Sundaramurthy, C., Dalton, D., [1993], «Corporate Governance Predictors of Adoption of Anti-takeover Amendments. An Empirical Analysis», Journal of Business Ethics, 12 (5), p. 371-378.

SHLEIFER, A., Vishny, R., [1986 a], «Large Shareholders and Corporate Control», Journal of Political Economy, 94 (3), p. 461-488.

SHLEIFER, A, VISHNY, R., [1986 b], «Greenmail, White Knights, and Shareholders Interests», The Rand Journal of Economics, 17 (3), p. 293-309.

TIROLE, J., [2006], « Takeovers ». In The Theory of Corporate Finance, Princeton University Press.

TURK, T., GOH, J., YBARRA, C., [2007], «The Effect of Takeover Defences on Long Term and Short Term Analysis' Earning Forecasts: the Case of Poison Pills», Corporate Ownership and Control,4 (4), p. 127-131.

\section{ANNEXES}

\section{Annexe A : Le calcul du nombre total des droits de vote, noté $\mathrm{C}$, qui sont exprimés lors des assemblées générales}

Les droits de vote à considérer sont ceux qui sont détenus par l'investisseur hostile, par les $\mathrm{K}$ membres du noyau dur qui possèdent plus de $\delta$ titres et par les $\mathrm{N}-\mathrm{K}$ membres du noyau dur qui possèdent moins de $\delta$ titres. Tous les autres actionnaires ne participent pas aux assemblées générales.

$$
\begin{gathered}
\mathrm{C}=\sum_{j} \mathrm{DV}_{j}=\mathrm{DV}_{h}+\sum_{j=1}^{\mathrm{K}} \mathrm{DV}_{j}+\sum_{j=\mathrm{K}+1}^{\mathrm{N}} \mathrm{DV}_{j} \\
=\gamma l_{h}+(1-\gamma) \delta+\sum_{j=1}^{\mathrm{K}}\left(\gamma l_{j}+(1-\gamma) \delta\right)+\sum_{j=\mathrm{K}+1}^{\mathrm{N}} l_{j} \text { d'après (1) }
\end{gathered}
$$




$$
\begin{gathered}
=\gamma l_{h}+(1-\gamma) \delta+\sum_{j=1}^{\mathrm{K}} \gamma l_{j}+\mathrm{K}(1-\gamma) \delta+\sum_{j=\mathrm{K}+1}^{\mathrm{N}} l_{j} \\
=\gamma l_{h}+(1-\gamma) \delta+\gamma(\mathrm{A}-\mathrm{B})+\mathrm{K}(1-\gamma) \delta+\mathrm{B} \\
=\gamma\left(l_{h}+\mathrm{A}\right)+(1-\gamma)(\delta+\delta \mathrm{K}+\mathrm{B})
\end{gathered}
$$

\section{Annexe B : La mise en évidence du seuil défensif}

$$
\begin{gathered}
\% \mathrm{DV}_{h}<\mathrm{S} \\
\gamma l_{h}+(1-\gamma) \delta<\mathrm{S}\left(\gamma\left(l_{h}+\mathrm{A}\right)+(1-\gamma)(\delta+\delta \mathrm{K}+\mathrm{B})\right) \\
\gamma l_{h}(1-\mathrm{S})<\mathrm{S} \gamma \mathrm{A}+(1-\gamma)(\mathrm{S} \delta-\delta+\mathrm{S} \delta \mathrm{K}+\mathrm{SB}) \\
l_{h}<\frac{\mathrm{S}}{1-\mathrm{S}} \mathrm{A}+\frac{(1-\gamma)}{\gamma(1-\mathrm{S})}(\delta(\mathrm{S}-1)+\mathrm{S} \delta \mathrm{K}+\mathrm{SB}) \\
\text { On note } \sigma_{\mathrm{S}}=\left(\frac{\mathrm{S}}{1-\mathrm{S}}\right)\left(\mathrm{A}+\left(\frac{1-\gamma}{\gamma}\right)\left(\frac{\delta(\mathrm{S}-1)}{\mathrm{S}}+\delta \mathrm{K}+\mathrm{B}\right)\right)
\end{gathered}
$$

On obtient $l_{h}<\sigma_{\mathrm{S}}$

Pour $\mathrm{S}=\frac{1}{2}$, on trouve $: l_{h}<\mathrm{A}+\frac{(1-\gamma)}{\gamma}(-\delta+\delta \mathrm{K}+\mathrm{B})$

Pour $\mathrm{S}=\frac{1}{2}$ et $\gamma=1$, on retrouve $: l_{h}<\mathrm{A}$ (condition nécessaire d'échec de l'OPA)

\section{Annexe C: La matrice hessienne}

A partir des expressions des dérivées partielles exprimées en (8) et (11), la matrice hessienne peut s'écrire :

$$
\left(\begin{array}{cc}
\frac{\partial^{2} \sigma_{S}}{\partial \delta^{2}}=0 & \frac{\partial^{2} \sigma_{S}}{\partial \delta \partial \gamma}=\left(\frac{-1}{\gamma^{2}}\right)\left(\frac{S}{1-S}\right)\left(\frac{S-1}{S}+K\right) \\
\frac{\partial^{2} \sigma_{S}}{\partial \gamma \partial \delta}=\left(\frac{-1}{\gamma^{2}}\right)\left(\frac{S}{1-S}\right)\left(\frac{S-1}{S}+K\right) & \frac{\partial^{2} \sigma_{S}}{\partial \gamma^{2}}=\left(\frac{2}{\gamma^{3}}\right)\left(\frac{S}{1-S}\right)\left(\frac{\delta(S-1)}{S}+\delta K+B\right)
\end{array}\right)
$$

\section{Annexe D : Preuve de la proposition (10) par récurrence}

La proposition (10) a déjà été établie au rang $1:$ si $\frac{1}{2} \leq \mathrm{S}<1, \delta=l_{1}$, et au rang 2 : si $\frac{1}{3} \leq \mathrm{S}<\frac{1}{2}, \delta=l_{2}$. Nous allons établir le cas général par récurrence. Supposons que la relation est vraie jusqu'au rang $k-1:$ si $\frac{1}{k} \leq \mathrm{S}<\frac{1}{k-1}$ alors $\delta=l_{k-1}$. Au rang $k-1$, la dernière situation d'étude correspond au cas $\left(S_{1}=\frac{1}{k}\right.$ et $\left.S=\frac{1}{k}\right)$. La dernière valeur de $\mathrm{S}_{1}$ est donc $\frac{1}{k}$ et la dernière valeur de $\mathrm{S}$ est également $\frac{1}{k}$.

Pour prolonger l'étude au rang $k$, nous considérons maintenant la situation : $\mathrm{S}_{1}=\frac{1}{k}$ avec $S<\frac{1}{k}$. Nous supposerons que la valeur de $S$ est supérieure ou égale à $\frac{1}{k+1}$. Nous sommes donc dans la situation $\mathrm{S}<\mathrm{S}_{1}$. Nous savons que dans cette situation la 
décroissance de $\delta$ implique un accroissement de $\sigma_{\mathrm{S}}$. Si le but du noyau dur est de garantir une valeur de $S$ strictement inférieure à $\frac{1}{k}$, alors le réglage $\delta=l_{k-1}$ n'est plus pertinent et il est nécessaire de diminuer $\delta$. Tant que sa valeur reste strictement inférieure à $l_{k}$, la décroissance de $\delta$ conduit à une augmentation de la valeur de $\sigma_{\mathrm{S}}$. Dès que $\delta$ atteint la valeur $l_{k}$, la valeur de $\mathrm{K}$ augmente d'une unité et devient ici $\mathrm{K}=k+1$. Dans ce nouvel état, nous avons donc $\mathrm{S}_{1}=\frac{1}{k+1}$.

Deux nouvelles situations doivent être étudiées : $\left(S_{1}=\frac{1}{k+1}\right.$ avec $\left.S=\frac{1}{k+1}\right)$ et $\left(S_{1}=\frac{1}{k+1}\right.$ avec $\left.\mathrm{S}<\frac{1}{k+1}\right)$. Si le but du noyau dur est de garantir une valeur de $\mathrm{S}$ strictement inférieure à $\frac{1}{k+1}$, nous sommes de nouveau dans la situation $S=S_{1}$ et la modification de $\delta$ est sans effet sur la valeur de $\sigma_{\mathrm{S}}$. Il n'est donc plus nécessaire de modifier la valeur de $\delta$. En réalité, un abaissement de $\delta$ jusqu'à la valeur $\delta=l_{k+1}$, n' a pas d'effet sur la valeur de $\sigma_{\mathrm{S}}$ mais renvoie une image négative au marché. Un abaissement de $\delta$ sous la valeur $l_{k+1}$ a pour effet d'augmenter la valeur de $\mathrm{K}$ d'une unité supplémentaire avec $\mathrm{K}=k+2$. Cela réduit immédiatement la valeur de $\mathrm{S}_{1}$, avec $S_{1}=\frac{1}{k+2}$. Avec cette nouvelle valeur de $S_{1}$, la variable $S$, qui est toujours égale à $\frac{1}{k+1}$, devient strictement supérieure à $S_{1}$. Cela conduit à une chute de la valeur de $\sigma_{\mathrm{S}}$ ce qui n'est pas souhaitable. Finalement, dans le cas où $\mathrm{S}_{1}=\frac{1}{k+1}$ avec $\mathrm{S}=\frac{1}{k+1}$, c'est donc bien $\delta=l_{k}$ qui constitue le meilleur réglage.

En conclusion, si $\frac{1}{k+1} \leq \mathrm{S}<\frac{1}{k}$, alors $\delta=l_{k}$ est le meilleur réglage. La proposition est donc vraie au rang $k$, ce qui achève la démonstration par récurrence. Il n'est pas nécessaire de s'intéresser au cas $\left(\mathrm{S}_{1}=\frac{1}{k+1}\right.$ avec $\left.\mathrm{S}<\frac{1}{k+1}\right)$ pour finir la démonstration car cette nouvelle situation n'intervient qu'au rang $k+1$. Si on note $\delta_{0}$ le meilleur réglage de $\delta$ nous pouvons affirmer que :

$$
\text { si } \frac{1}{k+1} \leq \mathrm{S}<\frac{1}{k}, \text { alors } \delta_{0}=l_{k}
$$

\section{Annexe E: L'optimisation de la valeur de gamma}

On suppose que $: \frac{1}{k+1} \leq \mathrm{S} \leq \frac{1}{k}$, ce qui implique $\delta=\delta_{0}=l_{k}$ et $\mathrm{K}=k$

L'inégalité $l_{h}<\sigma_{\mathrm{S}}$ devient alors

$$
\begin{gathered}
\gamma l_{h}+(1-\gamma) \delta_{0}<\mathrm{S}\left(\gamma\left(l_{h}+\mathrm{A}\right)+(1-\gamma)\left(\delta_{0}+k \delta_{0}+\mathrm{B}\right)\right) \\
\gamma\left(l_{h}(1-\mathrm{S})+\delta_{0}(\mathrm{~S}(1+k)-1)-\mathrm{SA}+\mathrm{SB}\right)<\delta_{0}(\mathrm{~S}(1+k)-1)+\mathrm{SB} \\
\gamma<\frac{\delta_{0}(\mathrm{~S}(1+k)-1)+\mathrm{SB}}{l_{h}(1-\mathrm{S})-\mathrm{SA}+\delta_{0}(\mathrm{~S}(1+k)-1)+\mathrm{SB}}=\gamma_{0}
\end{gathered}
$$


Pour $\mathrm{S}=\frac{1}{k+1}$, on obtient $\mathrm{S}(1+k)-1=0$ et l'inégalité devient alors :

$$
\gamma<\frac{\mathrm{SB}}{l_{h}(1-\mathrm{S})-\mathrm{SA}+\mathrm{SB}}
$$

\section{Annexe F : La contrainte d'obtention du quorum}

Si l'investisseur hostile participe aux assemblées alors $\mathrm{Q}=\mathrm{Q}_{h+}$ et la contrainte s'écrit :

$$
\begin{aligned}
& \mathrm{Q}_{h+} \geq \frac{1}{q} \\
& \frac{\mathrm{C}}{\mathrm{T}-\left(l_{h}+\mathrm{A}\right)+\mathrm{C}} \geq \frac{1}{q} \\
& \frac{\gamma\left(l_{h}+\mathrm{A}\right)+(1-\gamma)(\delta+\delta \mathrm{K}+\mathrm{B})}{\mathrm{T}-(1-\gamma)\left(l_{h}+\mathrm{A}-\delta-\delta \mathrm{K}-\mathrm{B}\right)} \geq \frac{1}{q} \\
& q \gamma\left(l_{h}+\mathrm{A}\right)+q(1-\gamma)(\delta+\delta \mathrm{K}+\mathrm{B}) \geq \mathrm{T}-(1-\gamma)\left(l_{h}+\mathrm{A}-\delta-\delta \mathrm{K}-\mathrm{B}\right) \\
& \gamma(q-1)\left(\mathrm{A}+l_{h}-\delta-\delta \mathrm{K}-\mathrm{B}\right) \geq \mathrm{T}-l_{h}-\mathrm{A}-(q-1) \delta-(q-1) \delta \mathrm{K}-(q-1) \mathrm{B} \\
& \gamma \geq \frac{\mathrm{T}-\mathrm{A}-l_{h}-(q-1)(\delta+\delta \mathrm{K}+\mathrm{B})}{q \mathrm{~A}+q l_{h}-\mathrm{A}-l_{h}-(q-1)(\delta+\delta \mathrm{K}+\mathrm{B})} \\
& \frac{\delta(\mathrm{S}(1+\mathrm{K})-1)+\mathrm{SB}}{\delta(\mathrm{S}(1+\mathrm{K})-1)+\mathrm{SB}+l_{h}(1-\mathrm{S})-\mathrm{SA}} \geq \frac{\mathrm{T}-\mathrm{A}-l_{h}-(q-1)(\delta+\delta \mathrm{K}+\mathrm{B})}{q \mathrm{~A}+q l_{h}-\mathrm{A}-l_{h}-(q-1)(\delta+\delta \mathrm{K}+\mathrm{B})} \\
& (\delta(\mathrm{S}(1+\mathrm{K})-1)+\mathrm{SB})\left(q \mathrm{~A}+q l_{h}+(.)\right) \geq(\delta(\mathrm{S}(1+\mathrm{K})-1)+\mathrm{SB})(\mathrm{T}+(.)) \\
& +\left(l_{h}(1-\mathrm{S})-\mathrm{SA}\right)\left(\mathrm{T}-\mathrm{A}-l_{h}-(q-1)(\delta+\delta \mathrm{K}+\mathrm{B})\right) \\
& (\delta(\mathrm{S}(1+\mathrm{K})-1))\left(q \mathrm{~A}+q l_{h}-\mathrm{T}\right)+(q-1) \delta\left(l_{h}(1-\mathrm{S})-\mathrm{SA}\right)(1+\mathrm{K}) \geq \mathrm{SB}\left(\mathrm{T}-q \mathrm{~A}-q l_{h}\right) \\
& +\left(l_{h}(1-\mathrm{S})-\mathrm{SA}\right)\left(\mathrm{T}-\mathrm{A}-l_{h}-(q-1) \mathrm{B}\right) \\
& \text { On pose }: \delta_{1}^{h+}=\frac{\left(l_{h}(1-\mathrm{S})-\mathrm{SA}\right)\left(\mathrm{T}-\mathrm{A}-l_{h}-(q-1) \mathrm{B}\right)+\mathrm{SB}\left(\mathrm{T}-q \mathrm{~A}-q l_{h}\right)}{((\mathrm{S}(1+\mathrm{K})-1))\left(q \mathrm{~A}+q l_{h}-\mathrm{T}\right)+(q-1)\left(l_{h}(1-\mathrm{S})-\mathrm{SA}\right)(1+\mathrm{K})} \\
& \text { On obtient : } \delta \geq \delta_{1}^{h+}
\end{aligned}
$$


On pose alors : $\delta_{1}^{h+}=\frac{r \mathrm{~B}+s}{-r \mathrm{~K}+t}$ avec $\left\{\begin{array}{l}r=\mathrm{S}\left(\mathrm{T}-q \mathrm{~A}-q l_{h}\right)+(q-1)\left(\mathrm{SA}-l_{h}(1-\mathrm{S})\right) \\ s=\left(\mathrm{T}-\mathrm{A}-l_{h}\right)\left(l_{h}(1-\mathrm{S})-\mathrm{SA}\right) \\ t=(\mathrm{S}-1)\left(q \mathrm{~A}+q l_{h}-\mathrm{T}\right)+(q-1)\left(l_{h}(1-\mathrm{S})-\mathrm{SA}\right)\end{array}\right.$

Si l'investisseur hostile ne participe pas aux assemblées, un calcul similaire au précédent conduit à :

$$
\delta \geq \delta_{1}^{h-}=\frac{\left(l_{h}(1-\mathrm{S})-\mathrm{SA}\right)\left(\mathrm{T}-\mathrm{A}-l_{h}-(q-1) \mathrm{B}\right)+\mathrm{SB}(\mathrm{T}-q \mathrm{~A})}{((\mathrm{S}(1+\mathrm{K})-1))(q \mathrm{~A}-\mathrm{T})+\left(l_{h}(1-\mathrm{S})-\mathrm{SA}\right)((q-1) \mathrm{K}-1)}
$$

On pose alors : $\delta_{1}^{h-}=\frac{u \mathrm{~B}+v}{-u \mathrm{~K}+w}$ avec $\left\{\begin{array}{l}u=\mathrm{S}(\mathrm{T}-q \mathrm{~A})+(q-1)\left(\mathrm{SA}-l_{h}(1-\mathrm{S})\right) \\ v=q=\left(\mathrm{T}-\mathrm{A}-l_{h}\right)\left(l_{h}(1-\mathrm{S})-\mathrm{SA}\right) \\ w=(\mathrm{S}-1)(q \mathrm{~A}-\mathrm{T})-(q-1)\left(l_{h}(1-\mathrm{S})-\mathrm{SA}\right)\end{array}\right.$

\section{Annexe G : Le seuil défensif en situation d'information incomplète}

$$
\begin{gathered}
\mathrm{E}\left(\% \mathrm{DV}_{h}\right)<\mathrm{S} \\
p_{(\delta, \gamma)}\left(\gamma l_{h}+(1-\gamma) \delta\right)<\mathrm{S}\left(\gamma\left(l_{h}+\mathrm{A}\right)+(1-\gamma)(\delta+\delta \mathrm{K}+\mathrm{B})\right) \\
\gamma l_{h}\left(p_{(\delta, \gamma)}-\mathrm{S}\right)<\mathrm{S} \gamma \mathrm{A}+(1-\gamma)\left(\mathrm{S} \delta-p_{(\delta, \gamma)} \delta+\mathrm{S} \delta \mathrm{K}+\mathrm{SB}\right) \\
\left.l_{h}<\frac{\mathrm{S}}{p_{(\delta, \gamma)}-\mathrm{S}} \mathrm{A}+\frac{(1-\gamma)}{\gamma\left(p_{(\delta, \gamma)}-\mathrm{S}\right)}\left(\delta\left(\mathrm{S}-p_{(\delta, \gamma)}\right)+\mathrm{S} \delta \mathrm{K}+\mathrm{SB}\right) \text { (on suppose } p_{(\delta, \gamma)} \neq \mathrm{S}\right) \\
\text { On note } \sigma_{\mathrm{S}}^{p}=\left(\frac{\mathrm{S}}{p_{(\delta, \gamma)}-\mathrm{S}}\right)\left(\mathrm{A}+\left(\frac{1-\gamma}{\gamma}\right)\left(\frac{\delta\left(\mathrm{S}-p_{(\delta, \gamma)}\right)}{\mathrm{S}}+\delta \mathrm{K}+\mathrm{B}\right)\right)
\end{gathered}
$$

On obtient $l_{h}<\sigma_{\mathrm{S}}^{p}$ 